In Cres. Vol. $4 N^{\circ}$ 2: pp. 305-316, 2013

\title{
EFECTO DE LA SALINIDAD EN EL CONTROL DE LA INTENSIDAD DE EPIBIOSIS DE LA CONCHA DE ABANICO*
}

\author{
EFFECT OF SALINITY IN CONTROLLING \\ EPIBIOSIS INTENSITY OF SCALLOPS
}

\author{
Eleuterio Encomendero ${ }^{1}$, Fernando Merino ${ }^{2}$, \\ Marcos Bocanegra ${ }^{3}$, Laura Rubio ${ }^{3}$
}

\section{RESUMEN}

Esta investigación tuvo como objetivos determinar el crecimiento de la concha de abanico, Argopecten purpuratus sometida a experimentación; determinar la diversidad de los epibiontes de la especie; determinar la mortalidad de la concha de abanico experimental; identificar la concentración de salinidad y tiempo de tratamiento adecuados para reducir los epibiontes con la menor mortalidad de los organismos cultivados. Se realizó un experimento factorial con tres concentraciones de salinidad aplicadas como baños por 5, 10 y 15 minutos para controlar los epibiontes. El crecimiento en peso de $A$. purpuratus varió entre $11,19 \mathrm{~g}$, como mínimo y 20,55 g como máximo. El crecimiento en altura valvar fluctuó entre 8.15 mm como mínimo y 11,9 mm como máximo. La composición de epibiontes de la concha de abanico estuvo dada por un reducido número de especies: Hydroides sp, Balanus, Semimitylus algosus, Aglaophenia sp. y Ciona intestinales, predominando Hydroides sp y Mitylus. La menor mortalidad de la concha de abanico se obtuvo a la salinidad de $20 \%{ }^{\circ}$ y la biomasa menor de epibiontes con el baño de 5 minutos. Se considera adecuado para reducir los epibiontes el tratamiento con $20 \%^{\circ}$ de salinidad y aplicado por 5 minutos.

PALABRAS ClAVE: Concha de abanico, Argopecten purpuratus, epibiosis, mortalidad.

* Recibido: 04 de junio del 2013; aprobado: 28 de noviembre del 2013.

1 Biólogo Pesquero. Magister en Ciencias del Mar. Profesor Principal, Universidad Nacional del Santa. Chimbote, Perú.

2 Biólogo pesquero. Maestro en Ciencias. Profesor Principal, Universidad Nacional del Santa. Chimbote, Perú.

3 Estudiantes de la Universidad Nacional del Santa. Chimbote, Perú. 


\begin{abstract}
This investigation's objectives were: determine the growth of the 'concha de abanico' Argopecten purpuratus under experimentation, determine the diversity of the epibionts of this species, determine the mortality of 'concha de abanico' under experimentation, identify the salinity concentration and the treatment time needed to reduce epibionts with the least mortality of the farmend organisms. Was carried out a factorial experiment with three concentrations of salinity applied as baths by 5,10 and 15 minutes to control the epibionts. The growth in weight of $A$. purpuratus have been between $11,19 \mathrm{~g}$ as minimun and $20,55 \mathrm{~g}$ as maximun. The growth in valve heigth has been between $8,15 \mathrm{~mm}$ as minimun and 11,9 $\mathrm{mm}$ at the maximun. The composition of the concha's de abanico epibionts was a reduced number of specia: Hydroides sp, Balanus, Semimitylus algosus, Aglaophenia sp. and Ciona intestinales, predominating Hydroides sp y Mitylus. The least concha de abanico`s mortality was obtained at $20 \%^{\circ}$ salinity and the least epibionts biomass was obtained with bath of 5 minutes. The salinity concentration of $20 \%^{\circ}$ applied by 5 minutes it is considered adequate to reduce epibionts at the minimun.
\end{abstract}

KEY WORDS: Concha de abanico, Argopecten purpuratus, epibiosis, mortality.

\title{
I. INTRODUCCIÓN
}

En el cultivo de concha de abanico Argopecten purpuratus influyen variables ambientales de carácter abiótico y biótico. Entre las primeras son importantes el fitoplancton, los parásitos y los organismos competidores componentes del macrofouling que afectan los cultivos de concha de abanico, ostra, Crassostrea gigas (Arakawa, 1990) y a otros organismos en cultivo.

El macrofouling es el conjunto de organismos que más afectan al cultivo de abanico. Es un grupo heterogéneo de organismos que se adhieren a los objetos sumergidos en el mar (Flimlin, 1987), incluyendo organismos marinos con estructuras duras. En el caso de la concha de abanico, el macrofouling se adhiere a la superficie externa de las valvas, edes, cabos y aros metálicos que forman las linternas de cultivo; y a los cabos y boyas que constituyen el sistema de flotación que sostiene las linternas en el agua, durante su cultivo. Al adherirse al sistema de cultivo, tales organismos pueden afectar a los organismos cultivados. Algunos compiten por alimento (Lesser y col. 1992), por ser filtradores igual que $A$. purpuratus. Otros compiten por oxígeno disuelto en el agua y otros son parásitos o epibiontes, como la ciona, poliquetos, balanus, choros, etc. El macrofouling y los epibiontes generan un trabajo adicional de limpieza y elevan el costo de producción durante las operaciones de desdoble, para dejar la semilla con el mínimo de macrofouling en las etapas de cultivo intermedio o final. 
El tratamiento del macrofouling para reducir sus efectos sobre el crecimiento y bienestar de los organismos en cultivo y minimizar el trabajo de limpieza se ha experimentado con baños de agua dulce o hidróxido de calcio (Gallo et al., 2004), o el uso de nivel bajo de clorinación y dióxido de cloro (Garrett y Laylor, 1995), con resultados alentadores. También se han usado agua a presión y raspado (Portillo, 2002); pero, en el último caso, el uso de mano de obra también es importante. Las experiencias citadas se ha realizado en otras latitudes, pero en Perú no hay antecedentes con concha de abanico, por lo que es importante experimentar para establecer tratamientos que apoyen la optimización de su cultivo.

Entonces se debe buscar otras alternativas más sanas para el medio ambiente marino, que controlen los epibiontes que usan a la concha de abanico como sustrato. Por ello hay que poner a prueba la variación de salinidad, de tal forma que aplicando baños de agua con diferentes concentraciones salinas se podría combatir las larvas de epibiontes, así como los epibiontes pequeños que se desarrollan sobre las valvas de la concha de abanico cultivada. Sin embargo, se desconoce la concentración salina adecuada que combata los epibiontes y genere la mínima mortalidad del organismo cultivado, así como el tiempo que debe durar el tratamiento con la concentración adecuada.

\section{PROBLEMA}

¿Cuál es la salinidad adecuada para controlar los epibiontes de la concha de abanico, Argopecten purpuratus cultivada, que genere la mínima mortalidad de los organismos en cultivo?

El conocimiento de tratamientos adecuados para reducir el crecimiento de los organismos componentes del macrofouling, que son epibiontes de la concha de abanico, puede reducir el costo de limpieza de la misma especie durante el desdoble. Esto, a su vez, generaría un cultivo más eficiente y mejorará las condiciones de cultivo de los organismos cultivados al reducir el peso sobre las valvas y la competencia por alimento, espacio y oxígeno de los epibiontes. Así se optimizaría el cultivo.

\section{OBJETIVOS}

Por ello los objetivos de esta investigación fueron: Determinar el crecimiento en peso y altura valvar de la concha de abanico; determinar la biomasa y diversidad del macroufling epibionte de la concha de abanico controlado por los 
baños con diferentes concentraciones salinas; determinar la mortalidad de la concha de abanico en cada tratamiento experimental; identificar la concentración salina y el tiempo de tratamiento, adecuados para reducir al máximo el macrofouling epibionte de la concha de abanico en cultivo, con la menor mortalidad de los organismos cultivados.

\section{MATERIALES Y MÉTODOS}

Se realizó un diseño experimental factorial. Se compararon varios grupos de organismos para determinar si las variables independientes afectaban significativamente a las variables dependientes de los organismos experimentales, constituidos por conchas de abanico.

La población de trabajo estuvo constituida por todas las conchas que tuvieron una altura valvar promedio de $42,02 \mathrm{~mm}$ y peso promedio de $11,51 \mathrm{~g}$. La población constó de 630 conchas de abanico distribuidas 50 en cada uno de los 12 pearl nets que se usaron para cada tratamiento, más 30 conchas de las que se tomaron los datos iniciales.

El trabajo comparó dos grupos de conchas de abanico. El primero, constituido por 30 conchas de abanico, representativas de aquellas que se sometieron a los tratamientos (600 animales). De ellas se obtuvieron: altura valvar y peso total e identificación y peso total de todos los epibiontes. El segundo grupo fue de 12 muestras de 30 animales provenientes de los 12 pearl nets del diseño experimental de trabajo. En total se muestrearon 360 conchas de abanico, considerando los 9 tratamientos y 3 grupos control. La experisencia se realizó en la concesión marina de las Empresa Acuacultura El Dorado SAC, ubicada en $09^{\circ} 11^{\prime} 31.3^{\prime \prime}$ latitud sur y 78 32'53.4" longitud oeste, en un área de $14 \mathrm{~m}$ de profundidad de la bahía de Samanco. En el lugar indicado se ubicaron 12 pearl nets, a una profundidad de $6 \mathrm{~m}$; ; en donde permanecieron un total de 71 días.

Para caracterizar el ambiente, se registró la temperatura y el oxígeno disuelto. En ambos casos, los registros se hicieron 2, 8 y $12 \mathrm{~m}$. de profundidad. Ambos parámetros se midieron con un oxímetro, que registra el oxígeno en mg. $\mathrm{L}^{-1}$ y la temperatura en ${ }^{\circ} \mathrm{C}$.

Por otro lado, los animales se midieron con un vernier de $25 \mathrm{~cm}$., registrando la altura valvar en $\mathrm{mm}$, aproximando al décimo de milímetro. El peso se obtuvo con una balanza analítica digital redondeando al décimo de gramo más cercano y a cada animal se le desprendió y pesó el total de epibiontes fijados en 
ambas valvas. Es decir, se tomó una muestra de 30 conchas de abanico para determinar las condiciones iniciales de los animales: altura valvar, peso total, mortalidad, biomasa de epibiontes. Antes de registrar las variables, los animales fueron tamizados para homogenizarlos en tamaño.

Antes de la siembra, se sometió a la concha de abanico homogenizada a baños, por 5,10 y 15 minutos en agua con salinidades de 20,25 y $30 \%^{\circ}$ de salinidad. Cada combinación de salinidad y tiempo de baño es un tratamiento, por lo que se tuvo 9 tratamientos.

En bandejas de 50 litros se preparó el agua de mar con salinidades de 20, 25 y $30 \%^{\circ}$, diluyendo agua de mar con salinidad de $35 \%^{\circ}$ con volúmenes previamente calculados de agua dulce. Así se obtuvieron 30 litros de agua de mar de cada una de las concentraciones propuestas. En las tres bandejas con las salinidades indicadas, más una bandeja con 30 litros de agua de mar, se colocaron tres pearl nets de color azul, con 50 conchas de abanico cada uno, los mismos que se fueron retirando de las bandejas cuando habían transcurrido 5, 10 y 15 minutos, respectivamente, para las diferentes concentraciones salinas. A los datos se les aplicó análisis de varianza, con el SPSS, versión 15.

\section{RESULTADOS}

La temperatura registrada a los 2,5 y $12 \mathrm{~m}$. de profundidad, varió en el período experimental entre $20.4{ }^{\circ} \mathrm{C}$ y $21.4{ }^{\circ} \mathrm{C}$ al inicio de febrero, lo cual da una variación de sólo $1^{\circ} \mathrm{C}$, en los $2 \mathrm{~m}$. de profundidad. En cambio, en el nivel de los $5 \mathrm{~m}$. de profundidad la temperatura varió entre $15.5^{\circ} \mathrm{C}$ y $20.7^{\circ} \mathrm{C}$, con una amplitud de variación de $5.2^{\circ} \mathrm{C}$. A los $12 \mathrm{~m}$. de profundidad, la variación de temperatura fue de $1.9^{\circ} \mathrm{C}$; es decir, una variación intermedia entre la variación de los 2 y la variación de los $5 \mathrm{~m}$. de profundidad.

El oxígeno, factor importante en la vida de la mayor parte de los seres vivos, varió entre $6.42 \mathrm{~mL} . \mathrm{L}^{-1}$ en enero y $9.25 \mathrm{~mL} . \mathrm{L}^{-1}$ en febrero, presentando una variación de $2.83 \mathrm{~mL} . \mathrm{L}^{-1}$; en cambio, en el nivel de los $5 \mathrm{~m}$. el oxígeno varió entre $0.78 \mathrm{~mL} . \mathrm{L}^{-1}$ en diciembre y $8.92 \mathrm{~mL} . \mathrm{L}^{-1}$ en febrero; con una variación importante de $8.12 \mathrm{~mL} . \mathrm{L}^{-1}$. Finalmente, en el nivel de los $12 \mathrm{~m}$. de profundidad, el oxígeno varió entre $0.16 \mathrm{~mL} . \mathrm{L}^{-1}$ y $2.75 \mathrm{~mL} . \mathrm{L}^{-1}$ con una variación total de $2.59 \mathrm{~mL} \cdot \mathrm{L}^{-1}$.

Hay que notar que tanto en temperatura como en oxígeno disuelto, las mayores variaciones se presentaron en $\operatorname{los} 5 \mathrm{~m}$. de profundidad y es en este nivel 
o cerca de él donde se instalan los cultivos de concha de abanico, por lo que se considera que los dos factores ambientales afectarían a los procesos biológicos de la especie en cultivo.

La experiencia duró 71 días, y se inició con animales de $42.02 \mathrm{~mm}$ de altura valvar promedio, que lograron una altura mínima promedio de $50.17 \mathrm{~mm}$ y máxima de $53.92 \mathrm{~mm}$. La altura valvar mínima se registró en el baño de $25 \%^{\circ}$ de salinidad por 15 minutos $(25 \mathrm{~S} 15 \mathrm{M})$ y la altura valvar promedio máxima se obtuvo en el baño de 5 minutos con agua con $30 \%^{\circ}$ (30S5M). En los demás tratamientos se registraron alturas valvares promedio intermedias entre los valores extremos ya indicados (Fig. 1).

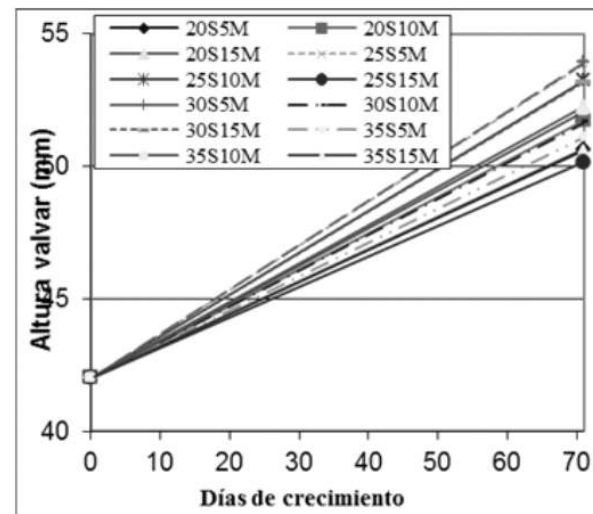

Figura 1. Crecimiento en altura valvar (mm)
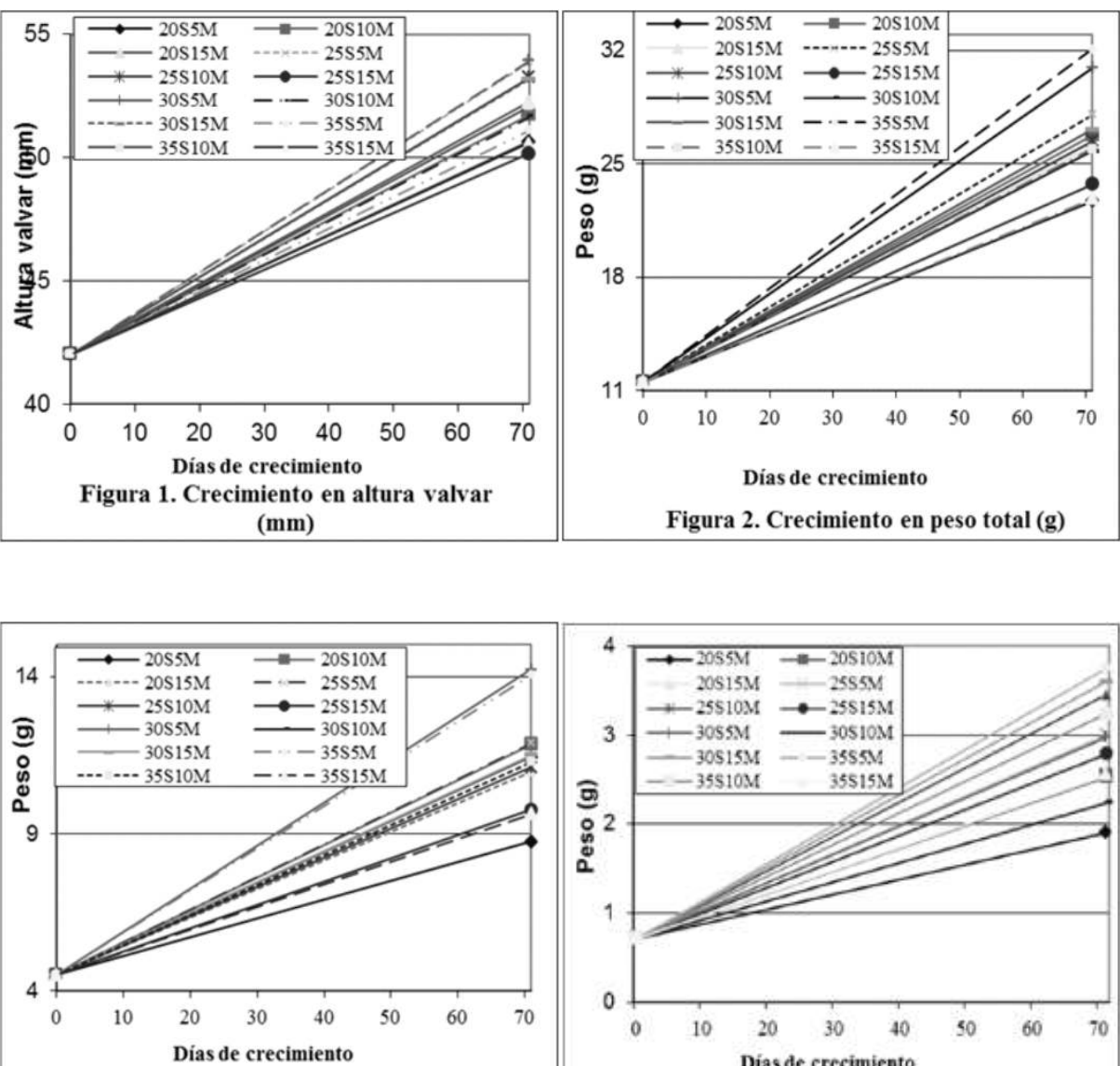

Figura 3. Variación del peso (g) de las partes blandas

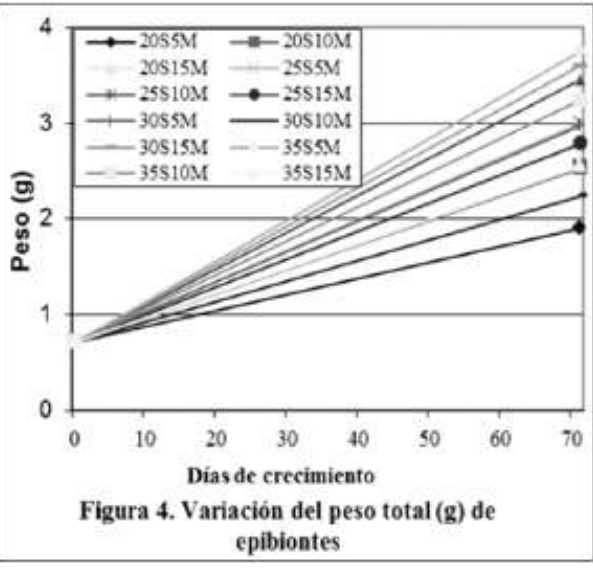


El peso inicial promedio de 30 conchas de abanico fue de $11.51 \mathrm{~g} \mathrm{y} \mathrm{al} \mathrm{cabo}$ de los 71 días experimentales se registró un peso promedio mínimo de $22.7 \mathrm{~g}$ (20S5M) y un peso promedio máximo de $32.06 \mathrm{~g}$ (35S5M). El peso mínimo se presentó en el tratamiento con $20 \%^{\circ}$ y por 5 minutos (20S5M). El peso promedio máximo se encontró en el tratamiento con $35 \%^{\circ}$ por 5 minutos $(35 \mathrm{~S} 5 \mathrm{M})$. Esto muestra que el crecimiento en el período de los 71 días varió entre $11.19 \mathrm{~g}$ como mínimo y 20.55 g como máximo (Fig. 2).

Las partes blandas de la concha de abanico, el talo y las gónadas, son las partes de mayor interés en el cultivo de la especie; por ello se han registrado sus pesos con el objeto de observar sus variaciones en relación con la presencia de epibiontes.

De acuerdo con la figura 3, el peso promedio de las partes blandas aumentó de $4.51 \mathrm{~g}$ al inicio de la experiencia, hasta $8.74 \mathrm{~g}$ como mínimo y hasta 14.21 g como máximo. El aumento mínimo se presentó en el tratamiento, en el que se dio el baño por 5 minutos y con salinidad de $20 \%^{\circ}$; y el incremento máximo se logró en el baño por 5 minutos con salinidad de $30 \%^{\circ}$. Sin embargo, no fue posible establecer una tendencia clara.

Respecto a la diversidad de los epibiontes, no es alta; pero las especies encontradas son organismos taxonómicamente diferentes. Las especies de epibiontes se encuentran listadas en la tabla 1.

Tabla 01

GRUPOS TAXONÓMICOS Y ESPECIES EPIBIONTES

DE LA CONCHA DE ABANICO

\begin{tabular}{ll}
\hline Grupo taxonómico & Especie \\
\hline Bivalvia & Semimitylus algosus \\
Polychaeta & Hydroides sp. \\
Hemichordata & Ciona intestinalis \\
Crustácea & Balanus laevis \\
Hidrozoa & Aglaophenia $s p$ \\
Brachiopoda & Discinisca lamellosa \\
\hline
\end{tabular}

En la muestra inicial, el peso promedio de los epibiontes fue de $0.72 \mathrm{~g}$. En cambio, al final los tratamientos presentaron diferentes pesos promedio de epibiontes. El menor peso de los epibiontes $(1.9 \mathrm{~g})$ se presentó en el tratamiento con salinidad de $20 \%^{\circ}$ durante 5 minutos y el mayor peso ( $3.75 \mathrm{~g}$ ) se registró en el tratamiento control con $35 \%^{\circ}$ de salinidad aplicado por 5 minutos. Pesos 
promedios de epibiontes relativamente altos también los presentaron el tratamiento con $20 \%^{\circ}$ de salinidad durante 15 minutos $(3.46 \mathrm{~g})$, con salinidad de $35 \%^{\circ}$ aplicado por 10 minutos ( $3.24 \mathrm{~g}$ ) y el tratamiento con $30 \%^{\circ}$ de salinidad aplicado por 15 minutos (3.6 g) (Figura 4).

Respecto a la mortalidad total, la más baja (19 animales) se dio en el tratamiento, que duró 10 minutos y la mortalidad mayor (22 animales), en el tratamiento, que duró 15 minutos. Sin embargo, en la figura 5 se puede observar que las diferencias son mínimas.

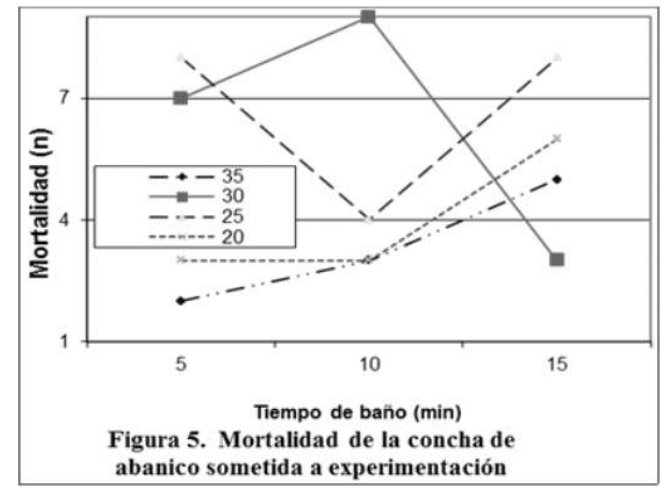

Respecto a la salinidad, las mayores mortalidades se presentaron en las salinidades de $30 \%^{\circ}$ ( 19 animales) y $25 \%^{\circ}$ (20 animales); las menores se presentaron en las salinidades de $35 \%^{\circ}$ (10 animales) y $20 \%^{\circ}$ (12 animales). En forma separada, el tratamiento que generó la más alta mortalidad fue el de $30 \%^{\circ}$ de salinidad aplicada durante 10 minutos ( 9 animales), pero el tratamiento de $25 \%^{\circ}$ de salinidad aplicado por 5 y 15 minutos generó una mortalidad simi$\operatorname{lar}$ (8 animales). La mortalidad total representa el $10.17 \%$ de los animales sometidos a experimentación (61 conchas de abanico de 600 iniciales).

\section{DISCUSIÓN}

Después de 71 de cultivo, luego de los tratamientos experimentales, el crecimiento valvar estuvo entre $8.15 \mathrm{~mm}$ y $11.87 \mathrm{~mm}$. En el primer caso, el crecimiento bajo se presentó en el tratamiento que duró 15 minutos con agua de $25 \%^{\circ}$ se presentó de salinidad; el crecimiento alto se presentó en la salinidad de $30 \%^{\circ}$, notándose diferencia también en la duración del baño, que en el último caso fue de sólo 5 minutos. Estos resultados muestran que es importante el tiem- 
po de tratamiento y de menor importancia, la concentración salina. A su vez, ello sugeriría que la concha de abanico soporta poco los cambios de salinidad y por períodos cortos. Lo afirmado anteriormente parece ser respaldado por crecimientos importantes en salinidades de $25 \%^{\circ}$ y duración de baño de 5 minutos (25S5M); sin embargo, también hay que hacer notar los crecimientos relativamente bajos en salinidades de $35 \%^{\circ}(35 \mathrm{~S} 5 \mathrm{M})$ y $(35 \mathrm{~S} 15 \mathrm{M})$, las cuales podrían estar relacionas con el manipuleo.

En el crecimiento en peso se nota mejor el efecto de los baños con salinidades y tiempos diferentes, ya que el crecimiento mínimo se presentó en la salinidad de $20 \%^{\circ}$; máximo se encontró en la salinidad de $33 \%^{\circ}$. En ambos casos con sólo 5 minutos de baño. Algo parecido ocurrió en el tratamiento con salinidad de $30 \%{ }^{\circ}$ y 5 minutos $(30 \mathrm{~S} 5 \mathrm{M})$.

Asimismo, se presentaron crecimientos relativamente bajos en peso, en aquellos casos que tuvieron crecimiento reducido en altura valvar (35S10M y $35 \mathrm{~S} 15 \mathrm{M})$. Es decir, el crecimiento mayor o menor se manifestó tanto en el crecimiento valvar como en el crecimiento en peso.

El crecimiento en esta variable investigada en la concha de abanico es coherente, tanto en el crecimiento en altura valvar como con el crecimiento en peso. El incremento mínimo en partes blandas se presentó en el baño que duró 5 minutos, pero con salinidad de $20 \%^{\circ}$ y el incremento máximo en el baño también por 5 minutos, pero con salinidad de $30 \%^{\circ}$.

El crecimiento de las partes blandas puede ser un buen indicador, tanto del crecimiento del animal en su conjunto como de la influencia que podrían tener los epibiontes.

Los epibiontes de la concha de abanico experimental presentaron poca diversidad, pues estuvo constituida por 6 especies de diferentes grupos taxonómicos como Semimitylus algosus (Bivalvia), Hydroides sp. (Polychaeta), Ciona intestinales (Hemichordata), Balanus laevis (Crustacea), Aglaophenia sp. (Hidrozoo) y Discinisca lamellosa (Brachiopoda). La cantidad de especies de epibiontes de la concha difiere de la cantidad de Bioincrustantes en estructuras de cultivo de $A$. purpuratus en la bahía Samanco. Pacheco y Garate (2005), repor$\tan 33$ especies como bioincrustantes en estructuras de cultivo de $A$. purpuratus en la bahía Samanco. Por tanto, existiendo una gran diversidad de organismos en el ambiente acuático de la concha de abanico, son pocos los que se fijan en las valvas de este animal cultivado en la bahía de Samanco. Los moluscos, tunicados, crustáceos y poliquetos son reportados también por Liñero \&. Siendo los 
poliquetos predominantes, estos pueden generar perjuicios por la mala presentación que dan a la media valva, cuando se comercializa en esta forma (Gallo y Col. 2004). Los poliquetos y otros epibiontes se fijan sobre las estructuras duras por su notoria rugosidad, que permite su colonización por la fauna sedentaria $\mathrm{o}$ sésil (Liñero \& Díaz, 2006).

El peso total inicial de los epibiontes fue en promedio $0.72 \mathrm{~g}$ y el final aumentó hasta un mínimo de $1.9 \mathrm{~g}$ y un máximo de $3.75 \mathrm{~g}$. En el primer caso, el peso de los epibiontes casi se triplicó y en el segundo caso superó al quíntuplo del peso inicial. Sin embargo, cuando se comparan epibiontes individuales, la mayoría de ellos: Semimitylus, Balanus, Aglohpenia y ciona, casi no estuvieron presentes al iniciar el trabajo experimental. Es probable que ello se deba a que a fines de noviembre tales especies todavía no habían iniciado sus procesos reproductivos y por ello, no había organismos de esas especies fijados en las valvas de la concha de abanico. Por tanto, los organismos epibiontes presentes al final de la etapa experimental no fueron aquellos que estuvieron cuando se aplicaron los baños, ya que eran pequeños y la densidad y diversidad fue mucho mayor.

Por otro lado, si hubieran sido epibiontes fijados antes de la aplicación de los baños, tendrían que haber sido epibiontes de mayor tamaño, como efectivamente, se presentaron algunos casos de balanus. De estas observaciones se desprende que la aplicación de los baños tendría que hacerse después de la fijación, con el objeto de matar los epibiontes fijados, antes que sigan desarrollándose y afectando a las conchas de abanico. También se reporta una relación significativa entre la presencia de poliquetos y la pobre supervivencia de de Crassostrea gigas (Gallo y Col. 2004).

Una de las preocupaciones de todo tratamiento que trata de eliminar a unos organismos y no afectar a otros, es la mortalidad. Con los baños en agua de diferente salinidad y diferentes tiempos de inmersión se quiere eliminar a los epibiontes fijados, afectando lo menos posible a la concha de abanico en cultivo.

En este caso, la mortalidad fue relativamente baja, que en conjunto de todos los tratamientos, apenas superó el $10 \%$, pero en cada uno de los tratamientos no superó el 2\%. En el tratamiento en el que se tuvo la mayor mortalidad ( 9 animales muertos, en $30 \mathrm{~S} 10 \mathrm{M})$, esta mortalidad no superó el $1.5 \%$. En el caso de las mortalidades menores de los otros tratamientos, ellas no superaron al $1 \%$ de los animales experimentales. Este es un valor importante asumiendo que se desea aplicar los tratamientos para controlar los epibiontes. 


\section{CONCLUSIONES}

El crecimiento en altura valvar de $A$. purpuratus, a partir de los $42.02 \mathrm{~mm}$, hasta $50.17 \mathrm{~mm}$ como mínimo y hasta $53.92 \mathrm{~mm}$ como máximo, significa que la concha de abanico creció como mínimo $8.15 \mathrm{~mm}$ y como máximo $11.9 \mathrm{~mm}$ en el período de 71 días.

La concha de abanico experimental creció, en promedio, entre $11.51 \mathrm{~g} \mathrm{a}$ 22.7 g como mínimo y entre 11.51 g a 32.06 g como máximo. Es decir 11.19 g como mínimo en 71 días y 20.55 g como máximo en el mismo período de tiempo. Los crecimientos son similares entre los animales tratados y no tratados, indicando que los tratamientos no tuvieron efectos significativos.

La diversidad de los epibiontes de la concha de abanico fue baja; estuvo constituida por Semimitylus algosus, Hydroides sp., Ciona intestinales, Balanus laevis, Aglaophenia sp y Discinisca lamellosa (Brachiopoda), las cuales en conjunto alcanzaron biomasas entre $1.9 \mathrm{~g}$ como mínimo y $3.75 \mathrm{~g}$ como máximo, predominando Semimitylus algosus, Hydroides sp., Ciona intestinales.

La mortalidad total de la concha de abanico por los tratamientos alcanzó a $10.17 \%$. Sin embargo, en cada tratamiento fue relativamente baja (entre 0.3 y $1.3 \%$ ) y similar a los grupos control, lo que sugiere que tal mortalidad sería debido principalmente a la manipulación.

La mortalidad menor de la concha de abanico se logró en la salinidad de $20 \%^{\mathrm{o}}$ y las biomasas menores de epibiontes se obtuvieron con los tiempos de baño de 5 minutos, por lo que el tratamiento de los epibiontes con salinidades de $20 \%^{\circ}$ por un tiempo de 5 minutos será la opción elegida para reducir al mínimo a los epibiontes.

\section{BIBLIOGRAFÍA CONSULTADA}

ARAKAWA, K. 1990. Competitors and fouling organisms in the hanging culture of the pacific oyster, Crassostrea gigas (Thumberg). Mar. Behav. Physiol, 17: 67-94.

Enright, C.; D. Krailo, L. Stapples; M. Smith; C. Vaughan; D. Ward; P. Gaul and E. BORGESE. 1983. Biological control of fouling algae in oyster aquaculture. Journal of Shellfish Research, 3(1):41-44.

FLIMLIN, G. AND G. MATHIS. 1987. Biological biofouling control in a field based nursery for the hard clam, Mercenaria mercenaria. World Aquaculture, 24 (4):47-48.

GAllo, M., M. García Y D. GodínEZ. 2004. Evaluación de dos tratamientos en la intensidad de gusanos poliquetos asociados a las valvas del ostión Crassotrea gigas (Thumberg, 1873). Ciencias Marinas, 30 (3): 455-464. 
GARRET, W. AND M. LAYLOR. 1995. The Effects of Low Level Chlorination and Chlorine Dioxide on Biofouling Control in a Once-through Service Water System. Procedings of The International Zebra Mussel and Other Aquatic Nuisance Organisms conference, Toronto, Canada, February $1995(133-142$.

LesSer, M., S. Shumway, T. CuCCI AND J. Smith. 1992. Impact of fouling organisms on mussel rope culture: interspecific competition for food among suspension-feeding invertebrates. J. Exp. Mar. Biol. Ecol., 165:91-102.

PACHECO, A y A. GARATE. 2005. Bioincrustantes en estructuras de cultivo de Argopecten purpuratus en bahía Samanco, Perú. Ecología Aplicada, 4(1,2).

PorTiLlo, E. 2002. Control biológico del fouling en tanques de cultivo de macroalgas mediante el gasterópodo Osilinus atratus (Wood, 1828). Bol. Int. Esp. Oceanogr. 18 (1-4): 401-404. 